\title{
THINKING 3D IN 2D: TEACHING THREE-DIMENSIONAL ANALYSIS AND ENGINEERING TECHNICAL WRITING ONLINE DURING COVID-19
}

\author{
George Lamont, Hamza Butt, Chimdindu Ohaegbu, Jessica Macri, Mayuri Punithan, and Steve Lambert \\ University of Waterloo \\ glamont@uwaterloo,steve@uwaterloo.ca
}

\begin{abstract}
Technical-communication skills impact several graduate attributes and practicing engineers widely attest that technical communication is a main professional activity, while employers have expressed concerns about students' lack of these skills. Engineering programs often teach technical communication with hands-on 3D activities. However, educators have faced challenges incorporating technical communication in online modes, compounded by a lack of consensus about sustainable delivery. Minecraft Education Edition has shown potential to teach STEM subjects and supplement engineering-design projects, but its use in post-secondary engineering education is only starting to receive serious attention. To address these challenges, we developed a Minecraft activity and observed its impacts on student engagement, learning, and perceptions of its relevance to engineering. Our results show that Minecraft is an effective and engaging platform to teach technical communication and has implications for designing relevant and sustainable online technical activities with easy group collaboration.
\end{abstract}

Keywords: engineering communication, technical writing, Minecraft, sustainable teaching, game-based learning.

\section{INTRODUCTION}

Of the $12 \mathrm{CEAB}$ graduate attributes, seven are directly impacted by students' technical-communication skills: problem analysis, design, teamwork, communication, professionalism, impact of engineering on society and the environment, and economics and project management. Engineering students express frustration with technical communication [18] and skepticism about technicalcommunication courses [2], but employers have voiced concern about gaps between academic preparation and practical communication in workplaces [17]. Overwhelming testimonials $[2,7]$ and survey data $[3,7,17$, $21]$ indicate that practicing engineers deem communication to be essential for success, more so than design, mechanics, manufacturing, economics, or computer skills [17] and helpful in professional advancement [2, 21]. Practicing engineers have reported spending between 19 and 23.3 hours per week producing their technical writing [18] and spending 14.9 to 19.6 hours responding to technical communication from others $[17,21]$, or more than half the workday $(64 \%)[2,3,17]$. Strong technical communicators are in high demand by technical disciplines $[4,20]$. As such, employers and engineers support more integration of communication skills into engineering programs [2, 21], and engineering students often express appreciation for these courses upon completion [2].

Engineering programs prepare students for professional technical communication in various pedagogical modalities. Many programs supplement core content courses with communication elements such as learning reflections, teach lab-report writing, or focus on linguistic conventions such as punctuation, style, and formatting [10]. Other programs require engineering students to take a standard introduction to academic writing. Another approach involves tailored engineering-communication courses, with more time and resources available for 3D spatial reasoning, and uses diverse 3D objects to facilitate investigation, process analysis, technical process writing, document design, and user-experience testing.

However, when engineering programs suddenly moved online to cope with COVID-19, educators had to relinquish these meaningful hands-on activities to teach technical communication. Replacing these activities sustainably online is difficult because the relevant pedagogical approaches are widely debated and there is a lack of research to describe how to transition them online. Technical communication resides at the core of engineering [25]. Many engineering documents follow structures found in research articles $[5,6,18]$, target practical implications [7], and involve complex technical matters through scientific syntaxes, descriptions, and classifications [5, 13, 28]. Engineering technical communication lessons often have students engage in hands-on three-dimensional activities before producing technical documents to be user-tested in person. These lessons require investments in objects such as puzzles or other complex objects and tools, all of which are subject to management complexity, loss, and breakage. Online, instructors often must settle for simpler tasks not requiring $3 \mathrm{D}$ objects or just removing this practical experience. 
Minecraft software can address these challenges effectively and sustainably and is already availablethough overlooked - at many institutions. Minecraft is an open-world sandbox game [8, 14, 16, 29], built with living ecology, working chemistry systems, and physics [8] analogous to the real world [9]. The game's creative mode enables players to build diverse designs with an endless supply of game items $[11,22]$. The education edition adds teacher controls to create focused learning environments $[11,16]$, interact with students through a central location [8], and use simple guides, a camera tool, and a code builder that enables students to use programming to perform tasks $[11,23]$. Minecraft is easy to modify and learn [24], allows construction of scenarios without programming experience, and enables intimate exploration of scenarios [14]. Student engagement with learning often increases $[8,16]$. Minecraft has been used to teach geometry, sustainable planning, literacy, informatics, coding, project management, chemistry, ecology, geography, biology, physics [1, 9, 24, 26, 29], Fibonacci spirals, a replica of working neurons [8], anatomical models, cell models, and engineering systems [1].

In engineering, Minecraft can supplement CAD models and physical miniatures [14] that students could complete prior to COVID-19. Educators can create realistic scenarios such as analyzing a defective machine [14], developing factory logistics, designing virtual vehicles, programming calculators or AI $[24,29]$, and building functioning computers (RAM, CPU, ALU, GPU) [8], drawbridges, advanced architecture [1], flying machines [9] and even lab-on-chip sensors then produced by a 3D printer [15]. This is due to Minecraft's "redstone" mechanic, which acts as the in-game electric circuitry system [8, 9]. Minecraft allows students to collaborate easily on projects [19], an observed weakness in graduate engineers [2, 4]. In 2019, Hobbes et al. used a Mann-U test to determine that of their 492 STEM students across 32 schools, mean scores on concept questions increased from $57.5 \%$ pre-activity to $88.0 \%$ post-intervention [16]. Students value game-based teaching. In 2020, Baek and colleagues' survey found that over $90 \%$ of university students believed game-based learning approaches result in more active and engaged results [29]. However, only a small body of work examines the educational use of Minecraft in post-secondary education [12], with most of it from K-12 classes [1, 9, 11, 19, 26]. Few cases explore Minecraft's use in professional engineering settings [8]; however, this is likely due to researchers' slow uptake $[9$, 15].

In this pedagogical reflection, we address the challenges of teaching technical communication online sustainably by reporting our teaching method with Minecraft and the effects on student engagement and learning outcomes. We developed complex 3D engineering simulations with coding elements that directed students to follow a technical manual to build a design, surveyed the students about their expectations before the task, directed students to perform the task, and then surveyed the students about their learning experience before directing them to complete their own technical manual.

Our results demonstrate that students are engaged by Minecraft-based technical-writing lessons, find the experience relevant to engineering, and learn meaningful lessons about technical writing in the process. These results have implications for online pedagogy, sustainable design of technical-writing pedagogy, discipline-specific task design in engineering communication, and collaborativedesign platforms in engineering education.

\section{METHODS: LESSON AND SURVEY DESIGN}

This pedagogical reflection observes existing teaching methods and only the changes required for online instruction because of the pandemic. It examines the work of 129 students in 4 sections of an introductory engineering-communication course in Civil Engineering (CivE) and Electrical and Computer Engineering (ECE) in Fall 2020, and Architectural Engineering (ArchE) and Management Engineering (MgtE) in Winter 2021. The reflection functions as pedagogical quality assurance, not experimentation with human subjects. No activity was adjusted to accommodate the reflection work, and no student was assigned to a control group. All reflection activity was conducted after tasks were completed and grades were issued. Students were not incentivized except for standard contributions points used for all course lessons. The technical-writing unit was presented in two week-long stages: (1) students followed a manual to build a structure in Minecraft with code from the perspective of the end-user of technical documentation, and (2) students acted as engineers by learning a lesson about technical documentation, developing a design, and creating technical documents for a third party to reproduce the design. The unit occurred in weeks 5 and 6 of a twelve-week course. The lesson was timed to refine students' technical-writing skills before producing a progress report and then a final project proposal. Our institution has provided all students and faculty full access to Minecraft Education Edition.

The first week's pre-survey asked students to rate their anticipated difficulty and engagement with the task, so that the students could reflect on an end user's attitudes before using technical documentation. Students were then required to build a three-storey structure according to exact specifications given in a manual produced before the course. Recreational Minecraft players generally prefer to place individual blocks of material, but we required students to use the code builder, which uses complex sequenced commands and Cartesian coordinates. The manual was designed to be generally correct but contain small flaws to expose students to the challenges faced by end-users of technical documentation. Students verified their completion of the task by taking four screenshots of 
the structure, including the student's "agent": a unique labelled avatar for the student administered by the software. In the post-survey, students rated their actual difficulty and engagement with the task. Additional written-response questions asked students to describe the provided manual's limitations, report what they had learned from the experience as end users of documentation, and explain whether they felt the Minecraft task was relevant to their engineering studies and why.

In the second week, students were taught a video lesson about fifteen fundamental principles of technical communication with various professional examples. In a new pre-survey, students rated their anticipated difficulty of completing the task and writing a technical manual for a non-expert end user, as well as their anticipated engagement with the task. Additional questions asked students to anticipate which strategies they would employ in their technical manuals.

Students then completed a second online 3D task. In Fall 2020, students were required to solve specific online Adobe Flash 3D puzzles and produce technical manuals for a third-party user. The simulation required students to deconstruct 3D puzzles consisting of multiple pieces that appear to interlock, but the pieces can be rotated, pulled, and pushed to create pathways to extricate each piece from the puzzle. The simulation allowed the user to rotate the puzzles freely in three dimensions and try manipulating pieces to experiment without penalty, but the pieces must be removed in a certain order. The simulation provided puzzles of increasing difficulty as the user progresses, numbering between 4 and about 15 pieces. Users were not penalized for errors but their time to solve each puzzle was displayed, leading to points awarded in the simulation for speedy resolution. We played through the simulation to identify which puzzle levels would present reasonable difficulty for the students to create a technical manual without an excessive time cost; levels below these were then given as practice to familiarize students with the simulation. The task was extremely focused because it had only one clear objective and one solution to accomplish it. Though its applicability to engineering was somewhat abstract, the task was relatively easy to define and assess.

Because Flash expired on Dec. 31st, 2020, we developed engineering simulations for student teams of up to five in Minecraft. This presented the opportunity to make more relevant simulations, but it required a large investment of resources to build them. For Management Engineering, we constructed an automated foodproduction factory with production-line inefficiencies, building-layout limitations, and safety problems. Students were required to identify these design flaws, design an upgrade for the factory, and produce a technical manual allowing an end user to upgrade an identical factory. Architectural Engineering students were required to construct the wall enclosure of a building they had studied in their studio design course or one from a list of buildings at our institution. Students then produced technical manuals for an end user to reproduce the same design. As such, these tasks were more open-ended and required students to define their own objectives. Finally, students completed a post-survey, which asked them to rate their experience, difficulty, engagement, and relevance of the task.

To investigate our course design's success in teaching communication skills, we examined students' perception of difficulty and their enjoyment of the first assignment. We also examined student perceptions of difficulty and efficacy in teaching technical writing skills for the second assignment. These questions allowed us to assess student engagement and the value of learning experiences. We computed the students' percentages for the pre- and postversions of the four selected questions. The data was examined on a class-by-class basis, while the efficacy data was also examined on a term-by-term basis. Thus, we further attempted to determine whether the students' academic programs influenced their engagement with the assignments and whether the switch from puzzles to Minecraft for the technical manual assignment impacted student experience.

\section{Results}

Our findings address the difficulty of teaching technical communication with 3D activities online and suggest that Minecraft stimulates student engagement, facilitates meaningful learning, and has considerable relevance for engineering students. For the first assignment, all students followed a technical manual to build a three-storey structure. We intended for students to follow instructions and experience challenges as end users. After reading a description of the task, students reported their anticipated difficulty; after the task, students reported their experienced difficulty. However, the results surprised us. Results were grouped as very difficult (VD), somewhat difficult (SD), neutral $(\mathrm{N})$, somewhat easy (SE), and very easy (VE).

Table 1: Perceptions of the difficulty of building in Minecraft

\begin{tabular}{|c|c|c|c|c|c|c|}
\hline \multirow{2}{*}{ Class } & Time & \multicolumn{5}{|c|}{ Percentage of Students / Option } \\
\cline { 3 - 7 } & & VD & SD & N & SE & VE \\
\hline CivE & Pre & 0.0 & 12.0 & 24.0 & 52.0 & 12.0 \\
\cline { 2 - 7 } F20 (25) & Post & 4.2 & 8.3 & 29.2 & 41.7 & 16.7 \\
\hline ECE & Pre & 0.0 & 8.3 & 36.1 & 30.6 & 25.0 \\
\cline { 2 - 7 } F20 (36) & Post & 2.8 & 11.1 & 30.6 & 41.7 & 13.9 \\
\hline Arch.E & Pre & 0.0 & 7.7 & 33.3 & 33.3 & 25.6 \\
\cline { 2 - 7 } W21 (39) & Post & 0.0 & 2.9 & 23.5 & 52.9 & 20.6 \\
\hline MgtE & Pre & 0.0 & 17.2 & 31.0 & 34.5 & 17.2 \\
\cline { 2 - 7 } W21 (29) & Post & 0.0 & 20.0 & 20.0 & 32.0 & 28.0 \\
\hline \multirow{3}{*}{ ToTAL } & Pre & 0.0 & 10.9 & 31.8 & 36.4 & 20.9 \\
\cline { 2 - 7 } & Post & 1.7 & 10.1 & 26.1 & 42.9 & 19.3 \\
\hline
\end{tabular}


Globally, students reported that the task was easier than they had anticipated. Individual courses presented more variety. At least $50 \%$ of the students in each program believed the assignment would be somewhat easy or very easy, and the percentage for the total population rose from 57.4 to $62.2 \%$ afterwards. More than half of the students were confident in their ability to complete the assignment, and many found their confidence justified. The percentage of students ranking the assignment somewhat difficult remained almost constant between the surveys, and changes in the "very difficult" category were minimal. Because the manual was long and had been designed with minor flaws, we anticipated that students would express more frustration and therefore higher experienced difficulty. We attribute these responses to the quality of the manual, which underwent several revisions for clarity to ensure that students could succeed. Some student comments suggested that a more flawed manual would allow students to better experience the frustrations of end users. Further, we did not establish the students' previous experience with Minecraft, which could have considerably affected the results here.

In contrast, the data regarding students' enjoyment of the assignment decreased, as seen in Table 2 below. Their responses were recorded as much less enjoyable (MLE), somewhat less enjoyable (SLE), neutral or about the same $(\mathrm{N} / \mathrm{S})$, somewhat more enjoyable (SME) or much more enjoyable (ME).

Table 2: Perceptions of the enjoyability of building in Minecraft

\begin{tabular}{|c|c|c|c|c|c|c|}
\hline \multirow{2}{*}{ Class } & \multirow{2}{*}{ Time } & \multicolumn{5}{|c|}{ Percentage of Students / Option } \\
\cline { 3 - 7 } & & MLE & SLE & N/S & SME & ME \\
\hline CivE & Pre & 0.0 & 0.0 & 0.0 & 36.0 & 64.0 \\
\cline { 2 - 7 } F20 (25) & Post & 4.2 & 8.3 & 25.0 & 41.7 & 20.8 \\
\hline ECE & Pre & 0.0 & 5.6 & 5.6 & 33.3 & 55.6 \\
\cline { 2 - 7 } F20 (36) & Post & 11.1 & 30.6 & 16.7 & 30.6 & 11.1 \\
\hline Arch.E & Pre & 0.0 & 5.1 & 12.8 & 43.6 & 38.5 \\
\cline { 2 - 7 } W21 (39) & Post & 5.9 & 2.9 & 29.4 & 44.1 & 17.7 \\
\hline MgtE & Pre & 3.5 & 0.0 & 6.9 & 44.8 & 44.8 \\
\cline { 2 - 7 } W21 (29) & Post & 4.0 & 16.0 & 12.0 & 52.0 & 16.0 \\
\hline \multirow{3}{*}{ TOTAL } & Pre & 0.8 & 3.1 & 7.0 & 39.5 & 49.6 \\
\cline { 2 - 7 } & Post & 6.7 & 15.1 & 21.0 & 41.2 & 16.0 \\
\hline
\end{tabular}

Over $80 \%$ in each class and $89.1 \%$ of students overall anticipated the task would be at least somewhat enjoyable, a result we anticipated because Minecraft has been a popular game in the past ten years. However, student enjoyment dropped after the task to $57.2 \%$ overall, with a minimum decrease of $20 \%$ for the individual classes. As well, the percentage of students who selected "somewhat less enjoyable" was initially $3.1 \%$ but rose to $15.1 \%$ on the post-survey. We anticipated these decreases in enjoyment because we intended for the students to experience the challenge of following instructions to complete a complex technical task. This experience was meant to stimulate the students to produce more rewarding experiences for their own end users in the next phase. $78.2 \%$ of the total population voted neutral or better on the post-survey, demonstrating that most students found building in Minecraft to be at least as enjoyable as their standard studies.

The students' written responses on the post-survey help clarify these apparent contradictions. While many students approved of the manual's simple-to-follow content, they wanted warnings for problems that caused several students to restart their buildings. Students also found the formatting confusing and reported losing their place in the steps. These added comments reveal that although students did not find the assignment itself difficult, they experienced document design that frustrated their attempts to use the technical documentation.

The second week of this technical-communication unit required students to write a technical manual explaining how to either 1) solve a Flash-based online puzzle in Fall 2020 or 2) complete an original design in Minecraft in Winter 2021. Students were to apply their own observations of the first assignment's instructions to their own manuals and consolidate what they had learned. They received a video lesson about technical-writing principles before completing the pre-survey, the task, and the postsurvey. Our goal was to observe the students' perceptions of technical writing after they knew our expectations but before their own attempts. Again, surveys asked students to rate their anticipated and then experienced levels of difficulty in writing a technical manual, as given in Table 3. Results were grouped into five categories: very difficult (VD), somewhat difficult (SD), neutral (N), somewhat easy (SE), and very easy (VE).

Table 3: Perceptions of the difficulty of writing a technical manual

\begin{tabular}{|c|c|c|c|c|c|c|}
\hline \multirow{2}{*}{ Class } & \multirow{2}{*}{ Time } & \multicolumn{5}{|c|}{ Percentage of Students / Option } \\
\cline { 3 - 7 } & & VD & SD & N & SE & VE \\
\hline CivE & Pre & 4.2 & 37.5 & 45.8 & 8.3 & 4.2 \\
\cline { 2 - 7 } F20 (25) & Post & 4.4 & 21.7 & 21.7 & 43.5 & 8.7 \\
\hline ECE & Pre & 2.9 & 60.0 & 20.0 & 14.3 & 2.9 \\
\cline { 2 - 7 } F20 (36) & Post & 3.1 & 28.1 & 28.1 & 34.4 & 6.3 \\
\hline Arch.E & Pre & 0.0 & 40.0 & 40.0 & 14.3 & 5.7 \\
\cline { 2 - 7 } W21 (39) & Post & 13.3 & 36.7 & 36.7 & 6.7 & 6.7 \\
\hline MgtE & Pre & 12.5 & 45.8 & 16.7 & 25.0 & 0.0 \\
\cline { 2 - 7 } W21 (29) & Post & 9.5 & 19.1 & 38.1 & 28.6 & 4.8 \\
\hline \multirow{3}{*}{ TOTAL } & Pre & 4.2 & 46.6 & 30.5 & 15.3 & 3.4 \\
\cline { 2 - 7 } & Post & 7.6 & 27.4 & 31.1 & 27.4 & 6.6 \\
\hline
\end{tabular}


In every class, the percentage of students who expected writing a manual to be somewhat or very easy was low, with a maximum $25 \%$ of students in individual classes and only $18.6 \%$ overall. However, a much larger percentage of the Fall 2020 students reported relative ease after the task than did their Winter 2021 peers. The civil-engineering students and electrical and computer-engineering students, who performed the Flash-based puzzle task, reported somewhat or very easy task completion at $52.2 \%$ and $40.6 \%$, respectively. However, only $33.3 \%$ of management-engineering and $13.3 \%$ of architecturalengineering students, who were required to create and document a complex Minecraft design, reported the task as somewhat or very easy. We believe these differences may result from the different task designs in the second week of the unit, but we did not investigate this difference because Flash-based platforms are no longer available. Despite these differences, the percentage of all students who ranked the task somewhat difficult decreased by $19.3 \%$ between the pre- and post-surveys, while those who described it as somewhat or very easy increased by $15.3 \%$. Additionally, $62.2 \%$ of the total population found the first assignment somewhat or very easy compared to $34.0 \%$ on the second assignment, suggesting that students found it more difficult to write instructions than to follow them.

Similar disparities were observed between the two terms of students when they were asked about the efficacy of the assignment in teaching them technical writing skills. Student responses were recorded as very ineffective (VI), somewhat ineffective (SI), neutral $(\mathrm{N})$, somewhat effective (SEf) or very effective (VEf).

Table 4: Efficacy of the assignment in teaching technical writing skills.

\begin{tabular}{|c|c|c|c|c|c|}
\hline \multirow{2}{*}{ Class } & \multicolumn{5}{|c|}{ Percentage of Students / Option } \\
\cline { 2 - 6 } & VI & SI & N & SEf & VEf \\
\hline $\begin{array}{c}\text { CivE } \\
\text { F20 (25) }\end{array}$ & 0.0 & 0.0 & 17.4 & 39.1 & 43.5 \\
\hline $\begin{array}{c}\text { ECE } \\
\text { F20 (36) }\end{array}$ & 3.1 & 0.0 & 9.4 & 46.9 & 40.6 \\
\hline $\begin{array}{c}\text { Total } \\
\text { F20 (61) }\end{array}$ & 1.8 & 0.0 & 12.7 & 43.6 & 41.8 \\
\hline $\begin{array}{c}\text { Arch.E } \\
\text { W21 (39) }\end{array}$ & 0.0 & 6.7 & 10.0 & 66.7 & 16.7 \\
\hline $\begin{array}{c}\text { MgtE } \\
\text { W21 (29) }\end{array}$ & 4.8 & 4.8 & 23.8 & 38.1 & 28.6 \\
\hline $\begin{array}{c}\text { Total } \\
\text { W21 (68) }\end{array}$ & 2.0 & 5.9 & 15.7 & 54.9 & 21.6 \\
\hline Total & 1.9 & 2.8 & 14.2 & 49.1 & 32.1 \\
\hline
\end{tabular}

In total, $81.1 \%$ of the students believed the activity was effective to some degree, while only $4.7 \%$ felt the activity was not effective. Noteworthy variation occurs between terms and courses. 41.8\% of Fall 2020 students regarded the activity as very effective at teaching technical writing skills, while only $21.6 \%$ of the Winter 2021 students did so, although a larger proportion (54.9\%) in Winter 2021 still regarded the activity as somewhat effective. We attribute this variation to the different tasks requiredmanuals for 3D puzzles in Fall 2020 vs. more complex Minecraft designs in Winter 2021, but we did not investigate this variation directly.

The efficacy of the assignment in teaching students technical writing skills was also assessed using the students' written responses on the surveys. On the pre- and post-surveys for the second task, many students said they would improve on the flaws of the original Minecraft manual with more white (unused) space, before-and-after images, and clearly delineated sections. In contrast, their commentary for the first assignment focused on content issues, confusing wording, and lack of images for specific build aspects, while comments about formatting were less specific. These results suggest that the tasks' experientiallearning design facilitated the students' learning of technical writing and their ability to articulate methods of clear technical communication.

Student perceptions of the relevance of the task provide additional insight into the utility of Minecraft in engineering-communication instruction. The post-survey of Week 5 asked students, "How relevant did you feel the use of Minecraft Education was to engineering studies and why?" Students provided written responses, which were analyzed and presented in Table 5 as very irrelevant (VIr), somewhat irrelevant (SIr), neutral or no comment (N/no), somewhat relevant (SR), or very relevant (VR).

Table 5: Student perceptions of relevance in engineering education.

\begin{tabular}{|c|c|c|c|c|c|}
\hline \multirow{2}{*}{ Class } & \multicolumn{6}{|c|}{ Percentage of Students / Option } \\
\cline { 2 - 6 } & VIr & SIr & N/no & SR & VR \\
\hline $\begin{array}{c}\text { CivE } \\
\text { F20 (25) }\end{array}$ & 4.0 & 0.0 & 12.0 & 40.0 & 44.0 \\
\hline $\begin{array}{c}\text { ECE } \\
\text { F20 (36) }\end{array}$ & 0.0 & 0.0 & 2.8 & 52.8 & 44.4 \\
\hline $\begin{array}{c}\text { Arch.E } \\
\text { W21 (39) }\end{array}$ & 0.0 & 5.7 & 11.4 & 71.4 & 11.4 \\
\hline $\begin{array}{c}\text { MgtE } \\
\text { W21 (29) }\end{array}$ & 0.0 & 0.0 & 4.0 & 44.0 & 52.0 \\
\hline Total & 0.8 & 1.7 & 7.5 & 54.6 & 37.0 \\
\hline
\end{tabular}

Across all four courses, $91.6 \%$ of students explicitly found Minecraft relevant to engineering education and their other coursework, while only $2.5 \%$ found the activity irrelevant to some degree. Four students did not respond to this question and were counted as "neutral/no comment." However, students in architectural engineering tended to find Minecraft "somewhat relevant" rather than "very relevant." We attribute this to the limitations of Minecraft (e.g. only partial physics and difficulty of producing curved structures) to simulate advanced architectural features and 
the task design, which could be refined in future iterations to better approximate authentic architectural-engineering activity.

\section{DISCUSSION}

We set out to observe whether Minecraft was a sustainable and effective method of teaching engineering technical communication online and whether students found the activities engaging, effective, and relevant. Our results suggest that Minecraft is a promising and versatile alternative to using physical 3D objects in hands-on technical communication tasks, and students perceive the platform as relevant to their studies.

Students were excited to attempt the Minecraft-based activities and enjoyed them. Even though the students knew that the task's purpose was to train their technicalcommunication skills, about which engineering students often express frustration [18, 27] and skepticism [2], 89.1\% of the students anticipated some enjoyment in comparison to their other studies. Students did not find the activity explicitly difficult, but enjoyment of the tasks fell to $57.15 \%$ after completion. This confirms research about younger students that converting a game to an educational outcome reduces students' perceptions of enjoyment, even when the activity itself is the same [19]. Our objective was to expose students to the challenges experienced by end users following flawed technical documentation. Our manual was less flawed than we intended, but future work can explore the effects of flawed vs. polished manuals on student enjoyment and engagement, and better establish students' baseline Minecraft skills. Still, student commentary revealed that students experienced frustration with document formatting and clarity, forcing some students to re-start their structures and possibly accounting for the decrease in enjoyment. Some students in all five streams of civil, electrical, computer, management, and architectural engineering commented in written-answer questions that they perceived considerable value of immersing them in this end-user experience.

Another reason for the decrease in enjoyment may be the need to create more discipline-specific cases for discrete streams of engineering students [27], whereas our first task required students to build a generic structure. Some students in each stream reported in commentary responses that they did not always see how this generic task applied to their fields, so instructors must strive to create tasks that interest students [20]. In Winter 2021, we developed the specific scenarios for Management Engineering (factory optimization) and Architectural Engineering (constructing a wall enclosure already studied in the studio course), but we did not ask students to rate their enjoyment of these tasks because those results were not easily comparable to the Fall 2020 puzzle task. Further research can explore more sophisticated stream-specific scenarios and observing student enjoyment.
Students also found the Minecraft-based technicalwriting unit effective at teaching technical communication in an engineering program. In commentary, some students in all streams reported that Minecraft has certain limitations, such as a focus on lines and right angles rather than curves, limited physics realism, and the basic nature of the game's code-builder feature. However, a large majority of students $(81.1 \%)$ found the activity effective for an engineering program, and less than $5 \%$ found it ineffective. Giving the students example documents to follow allows them to critically examine the communications they must eventually produce [13] and recognize the procedures for creating technical documentation [4]. In commentary, students frequently commented that experiencing the process of using and then writing technical documents taught them to consider their multiple audiences in engineering practice and improve their clarity and accessibility, which are key learning outcomes for engineering students $[3,6]$.

More generally, students appeared to distinguish between their experienced enjoyment of a particular task and the platform's relevance to engineering education. $91.6 \%$ of students rated the platform as relevant, while only $2.5 \%$ saw the platform as irrelevant. In commentaries, several students admitted to initial skepticism about using Minecraft but discovered tremendous potential for engineering scenarios beyond technical communication. Several students commented that it was the most "handson" experience of this term, which has been taught online during the COVID-19 pandemic. This supports research that Minecraft can provide very realistic engineering scenarios [9] and offer advantages over some commercial CAD programs and other software because it is faster to use, more affordable [16, 19, 24], and allows instantaneous collaboration [15]. Minecraft's simple learning curve and ease of collaboration $[14,17]$ make it a versatile platform to develop these lessons if educators can invest in learning the software and adapting teaching methods [19]. Minecraft activities are in many ways far more sustainable than traditional teaching approaches: Minecraft eliminates the need for real 3D objects, reduces expense and maintenance, minimizes setup and tear-down time, allows easy group collaboration and inclusion, and is scalable to very large classes. Minecraft Education Edition has a maximum server size of 30 , requiring instructors to create workgroups no larger than 30 . Though we provided a hosted server, we found that student groups often preferred to create their own servers and work on their own. The use of Minecraft does not automatically make a lesson engaging, and it presents costs to the instructor to learn the software and develop meaningful simulations. Future research can investigate methods to develop and share meaningful simulations to reduce development costs. 


\section{CONCLUSION}

This pedagogical reflection presents compelling evidence that Minecraft is an effective and engaging medium of teaching engineering technical communication sustainably and allows instructors to conduct meaningful, practical technical-communication activities online. We presented technical simulations far more complex and engineering-specific than we achieved in the classroom, and for less cost and with more flexibility for students to use the simulation when they had time. Our findings demonstrate that students remained engaged even after they encountered difficulties in following manuals and that students found it easier to follow instructions than to write them. We observed that students generally recognized the difficulties faced by end users of documentation and transferred that experience to their document creation. We observed that stream-specific scenarios are essential to generating student buy-in but also that students found Minecraft an effective and relevant medium to teach engineering technical communication even when they found limitations in individual tasks. Our findings have dramatic implications for how engineering educators can teach technical communication at lower cost, with less waste, and with less equipment-administration loads.

Future work should explore several unanswered questions and design issues so far. The first assignment includes documentation flaws intended to immerse students in the experience of using others' technical documents, but instructions for this task should include a warning so that students understand that the technical documentation, not the student, may be the source of some difficulty. Future work could consider how to evaluate whether students transfer observations as end users significantly into their technical writing in communications lessons. Future research could also explore whether students carry these lessons into their engineering-content courses and over time, with ethics approval. Future research can further investigate why students enjoy various Minecraft simulations and consider how to make scenario design sustainable for educators to mitigate the costs of developing stream-specific simulations. Finally, future work can explore whether Minecraft's simple collaboration apparatus impacts students' feelings of inclusion and belonging in their cohorts.

\section{ACKNOWLEDGMENTS}

We would like to acknowledge the extensive technical contributions of Dmitrii Tcyrenov and Mellisa Lu, who developed the highly innovative first iteration of the production factory, which was an extremely complex task, and without which this project would not have succeeded. We also want to thank Raymond Ho, Ryan Bromley, Romaisa Ahmed, Dustin Kleiboer, Jogita Mahi, Cecilia Martin, and Haidar Mashlab for their technical assistance in refining and expanding the Minecraft factory simulation used in Winter 2021 for Management-Engineering students. Their work enlarged the factory to add a local distribution system and interactions with a simulated market, which has tremendous potential for even more expansion.

\section{References}

[1] Anton Petrov, Using Minecraft in Education: A Qualitative Study on Benefits and Challenges of Game-Based Education. Toronto, ON; Ontario Institute of Studies in Education, 2014, 98 pp.

[2] Beverly Coulter, Roslyn Petelin, Justine Gannon, Kate O'Brien, and Corrie Macdonald, "Enhancing technical writing skills for undergraduate students," in Australian Association for Engineering Education Conf., AAEE2017, Nazmul Huda, David Inglis, Nicholas Tse and Graham Town (ed.) (Sydney, AU; 10-13December 2017), 9 pp., 2017.

[3] C. Heylen and J. Van der Sloten, "A technical writing program implemented in a first year engineering design course at Ku Leuven," in European Society for Engineering Education, SEFI2012, (Thessaloniki, EL; 23-26September 2012), 2 pp., 2012.

[4] Carolyn Boiarsky, "Teaching engineering students to communicate effectively: A metacognitive approach," Int. J. Engng. Ed., vol. 20, no. 2, pp. 251-260, 2004. Available as of January, 2004, from https://www.researchgate.net/publication/228861996 Teac hing_Engineering_Students_to

Communicate_Effectively_A_Metacognitive_Approach

[5] Cheryl Bodnar and Jennifer Kadlowec, Initial Validation of a Technical Writing Rubric For Engineering Design, International Journal of Engineering Pedagogy, vol. 8, no. 1, pp. 81-91, 2018.

[6] David N. Dobrin, "What's difficult about teaching technical writing," College English, vol. 44, no. 2, pp. 135-140, February 1982.

[7] Dorothy A. Winsor, "Engineering Writing/Writing Engineering," College English, vol. 41, no. 1, pp. 58-70, February 1990.

[8] Glenn Ekaptura, Charles Lin, and Kho I. Eng, "Minecraft: A game as an education and scientific learning tool," in Information Systems International Conf., ISICO2013, (Bali, IN; 2-4December 2013), 6pp., 2013.

[9] H. Chad Lane and Sherry Yi, Playing with virtual blocks: Minecraft as a learning environment for practice and research, Champaign, IL: Illinois Informatics Institute, 2017, pp. 145-166.

[10] Jo Allen, "The case against defining technical writing," Journal of Business and Technical Communication, vol. 4, no. 2, pp. 68-77, September 1990.

[11] John Miller and Chris Fornell Scott, Unofficial Minecraft STEMLAB for Kids. Beverly, MA: Quarto Knows, 2018, 140 pp. \{ISBN: 978-1631594830\}

[12] Joy Egbert and Nataliia Borysenko, "Standards, engagement, and Minecraft: Optimizing experiences in language teacher education," Teaching and Teacher Education, vol. 85, pp. 115-124, 2019. Available as of October,

2019 ,

from 
https://www.researchgate.net/publication/336183544_Stand ards_engagement_and

Minecraft Optimizing experiences in language teacher e ducation

[13] Joy L. Colwell, Jana Whittington, and Carl F Jenks, "Writing challenges for graduate students in engineering and technology," in ASEE Annual Conference \& Exposition, ASEE11, (Vancouver, BC; 26-29June 2011), 13 pp., 2011.

[14] Katharina Schuster, Kerstin Grob, Anka Simone Richert, Rene Vossen, and Sabina Jeschke, "Preparing for industry 4.0 - collaborative virtual environments in engineering education," in The International Conference on E-Learning in the Workplace, ICELW15, (New York, NY; 10-12June 2015), 6 pp., 2015.

[15] Kyukwang Kim, Hyeongkeun Kim, Seunggyu Kim, and Jessie S. Jeon, "MineLoC: A rapid production of a Lab-ona-Chip biosensors using 3D printer and the sandbox game, Minecraft," Sensors (Basel), vol. 18, no. 6, June 2018. Available as of June 10, 2018, from https://www.mdpi.com/303306

[16] Laura Hobbs, Carly Stevens, Jackie Hartley, Mark Ashby, Benjamin Jackson, Lauren Bowden, Jordan Bibby, and Sophie Bentley, "Science hunters: Teaching science concepts in schools using Minecraft," Action Research and Innovation in Science Education, vol. 2, no. 2, pp. 13-21, 2019.

Available as of November 21, 2019, from https://uwerepository.worktribe.com/output/2976984

[17] Laura Reave, "Technical communication instruction in engineering schools: A survey of top-ranked U.S. and Canadian programs," Journal of Business and Technical Communication, vol. 18, no. 4, pp. 452-490, October 2004. Available as of October, 2004, from https://www.researchgate.net/publication/240709572_Tech nical_Communication Instruction in Engineering SchoolsA Survey of TopRanked_US_and_Canadian_Programs

[18] Marcia Marten Pierson and Bion L. Pierson, "Beginnings and endings: Keys to better engineering technical writing," IEEE Transactions on Professional Communication, vol. 40, no. 4, pp. 299-304, January 1998.

[19] Maria Cipollone, Catherine Schifter, and Rick A. Moffat, "Minecraft as a creative tool: A case study," International Journal of Game-Based Learning, vol. 4, no. 2, pp. 1-14, April 2014. Available as of April, 2014, from https://www.researchgate.net/publication/287093943_Mine craft_as_a_Creative_Tool_A_Case_Study

[20] Melanie I. Cashin, Developing global literacy: Best practices for teaching technical communication internationally. Mankato, MN: Minnesota State University, 2011, 258 pp.

[21] Richard M. Davis, Technical writing: Its Importance in the Engineering Profession and Its Place in Engineering Curricula-A Survey of the Experience and Opinions of Prominent Engineers. Technical report No. 75-5. ERIC Institute of Education Sciences, 1975. $84 \mathrm{pp}$.

[22] Sandra Bebbington and Andre Vellino, "Can playing Minecraft improve teenagers' information literacy?," Journal of Information Literacy, vol. 9, no. 2, pp. 6-26, December 2015. Available as of December, 2015, from https://ojs.lboro.ac.uk/JIL/article/view/PRA-V9-I2-1/2245
[23] Simon Baars and Sander Meester, "CodeArena: Inspecting and improving code quality metrics in Java using Minecraft," EasyChair Preprint, no. 734, January 2019. Available as of January 18, 2019, from https://easychair.org/publications/preprint/C8hB

[24] Steve Nebel, Sascha Schneider, and Gunter Daniel Rey, "Mining learning and crafting scientific experiments: A literature review on the use of Minecraft in education and research," Educational Technology \& Society, vol. 19, no. 2, pp. 355-366, $\quad$ September 2015. Available as of January, 2016, from https://www.researchgate.net/publication/301232882_Mini ng_Learning_and_Crafting

Scientific_Experiments_A_Literature_Review_on_the_Use of_Minecraft_in_Education_and_Research

[25] Steven M. Gerson, Writing That Works: A Teacher's Guide to Technical Writing. Topeka, KS: Kansas Curriculum Center, 2015, $100 \mathrm{pp}$.

[26] Thorkild Hanghoj, Heidi Hautopp, Casten Jessen, and Rikke Chistoffersen Denning, "Redesigning and reframing educational scenarios for Minecraft within mother tongue education," in European Conference of Game Based Learning, ECGBL14, Carsten Busch (ed.) (Berlin, DE; 910October 2014), 8 pp., 2014.

[27] Tracy Bridgeford, Karla Saari Kitalong, and Richard Selfe, Innovative Approaches to Teaching Technical Communication. Logan, UT: Utah State University Press, 2004, $147 \mathrm{pp}$.

[28] W. Earl Britton, "What is technical writing?," College Composition and Communication, vol. 16, no. 2, pp. 113 116, May, 1965.

[29] Youngkyun Baek, Ellen Min, and Seongchul Yun, "Mining educational implications of Minecraft," Computer in the Schools, vol. 37, no. 1, pp. 1-16, February 2020 Available as of February 12, 2020, from https://www.researchgate.net/publication/339234415 Mini ng_Educational_Implications_of_Minecraft 